\title{
Milliarcsecond angular resolution of reddened stellar sources in the vicinity of the Galactic center ${ }^{\star}$
}

\author{
A. Richichi ${ }^{1}$, O. Fors ${ }^{2,3}$, E. Mason ${ }^{4}$, J. Stegmeier ${ }^{1}$, and T. Chandrasekhar ${ }^{5}$ \\ ${ }^{1}$ European Southern Observatory, Karl-Schwarzschild-Str. 2, 85748 Garching bei München, Germany \\ e-mail: arichich@eso.org \\ 2 Departament d'Astronomia i Meteorologia, Universitat de Barcelona, Martí i Franqués 1, 08028 Barcelona, Spain \\ 3 Observatori Fabra, Camí de l'Observatori s/n, 08035 Barcelona, Spain \\ ${ }^{4}$ European Southern Observatory, Santiago, Chile \\ 5 Physical Research Laboratory, 380009 Ahmedabad, India
}

Received 2 June 2008 / Accepted 13 June 2008

\begin{abstract}
Aims. For the first time, the lunar occultation technique has been employed on a very large telescope in the near-IR with the aim of systematically achieving milliarcsecond resolution on stellar sources.

Methods. We have demonstrated the burst mode of the ISAAC instrument, using a fast read-out on a small area of the detector to record many tens of seconds of data at a time on fields of a few squared arcseconds. We used the opportunity to record a large number of LO events during a passage of the Moon close to the Galactic center in March 2006. We developed and employed for the first time a data pipeline for the treatment of LO data, including the automated estimation of the main data analysis parameters using a wavelet-based method and the preliminary fitting and plotting of all light curves.

Results. We recorded $51 \mathrm{LO}$ events over about four hours. Of these, 30 resulted in sufficient quality to enable a detailed fitting. We detected two binaries with subarcsecond projected separation and three stars with a marginally resolved angular diameter of about 2 milliarcsec. Two more stars, which are cross-identified with SiO masers, were found to be resolved and in one case we could recover the brightness profile of the extended emission, which is consistent with an optically thin shell. The remaining unresolved stars were used to characterize the performance of the method.

Conclusions. The LO technique at a very large telescope is a powerful and efficient method for achieving angular resolution, sensitivity, and dynamic range that are among the best possible today with any technique. The selection of targets is naturally limited and LOs are fixed-time events; however, each observation requires only a few minutes, including overheads. As such, LOs are ideally suited to filling small gaps during the idle time between standard observations.
\end{abstract}

Key words. techniques: high angular resolution - astrometry - occultations - binaries: general - masers

\section{Introduction}

The method of observing lunar occultations (LO) of background stars to derive their angular diameter, as well as other characteristics such as binarity, has been employed for decades and provides angular resolution at the milliarcsecond (mas) level independently of the diameter of the telescope used. This surpasses the diffraction limit of even the largest single telescopes and rivals the resolution of long-baseline interferometry (LBI), even with baselines of hundreds of meters. Basically, the LO technique relies on the lunar limb as a diffracting edge, rather than on the diameter of the telescope. The diffraction fringes are generated in space, and due to their relatively fast motion over the telescope, they must be sampled at rates of about $0.1-1 \mathrm{kHz}$. Combined together, these factors greatly diminish the adverse effects of atmospheric turbulence, which are the main limit of other high angular resolution techniques. Also, LO permit not only a model-dependent derivation of source parameters, such as the angular diameter and binary parameters, but also a model-independent reconstruction of the brightness profile of the source according to maximum-likelihood, or even a unique

\footnotetext{
^ Based on observations made with ESO telescopes at Paranal Observatory.
}

reconstruction by light curve inversion in special cases. The time required for observation (dominated by overheads, since an occultation lasts much less than $1 \mathrm{~s}$ ) and for data analysis is significantly shorter than for most other high angular resolution methods.

Of course, LO suffer from a number of significant drawbacks, and first among them is that the sources cannot be chosen at will. The Moon covers only about $10 \%$ of the celestial sphere in its apparent orbit. Lunar occultations are fixed time events, and as such subject to weather and instrumental downtimes. Finally, the lunar limb only provides a 1D scan of the source, although observations from different sites, or at different epochs, can in principle be combined under favorable circumstances. Due mainly to the chromatic properties of the scattered light background around the Moon and of the diffraction fringes, the near-IR is ideally suited to observing LO. More details on the method, its performance, the data analysis, and the results can be found in Richichi $(1985,1996)$ and Fors (2006). The CHARM2 catalog alone (Richichi et al. 2005) lists 1815 LO results in the field of high angular resolution.

While the angular resolution of LO is mainly set by the lunar limb rather than by telescope size, this parameter obviously plays a crucial role in determining the limiting sensitivity. Richichi (1997) studied the performance of LO under a number 


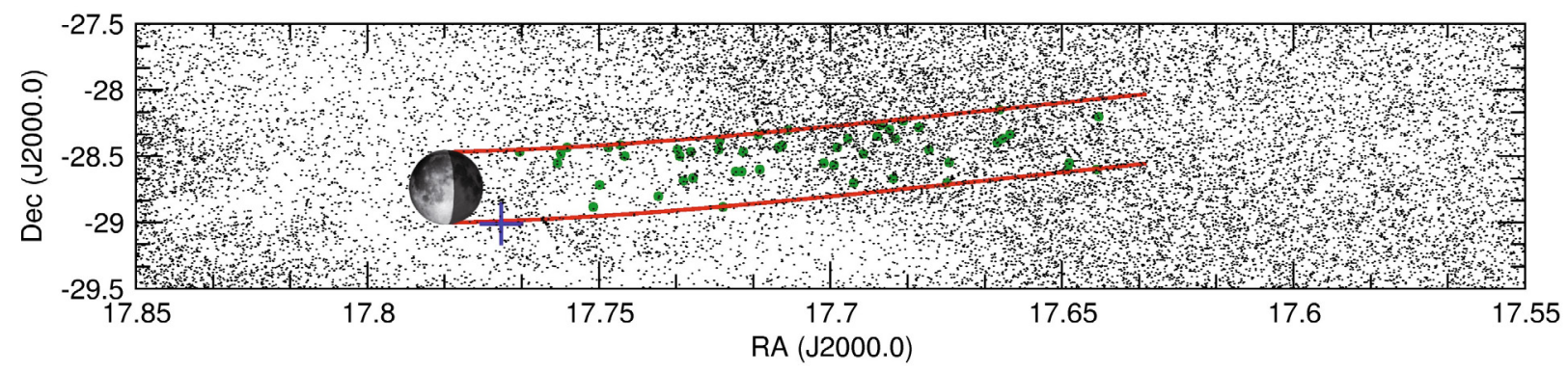

Fig. 1. The area close to the sources occulted by the Moon in the night of March 21, 2006. The small dots represent sources in the 2MASS catalog with magnitude $K \leq 7.5$. The heavier dots are the sources for which we could record a lunar occultation. The apparent path of the Moon, moving from West to East, is also shown. The cross marks the Galactic center.

of circumstances, including the then-futuristic use of IR array detectors on an $8-\mathrm{m}$ class telescope. This has recently become reality: Richichi et al. (2006b) and Fors et al. (2006, 2008) reported in a preliminary form on the use of the ISAAC instrument in the so-called burst mode at the ESO VLT $8.2 \mathrm{~m} \mathrm{Antu}$ telescope.

In the present paper, we provide a detailed account of the observations carried out during a few hours in the night of March 21, 2006, when 51 LO events were recorded. A second batch of observations was carried out in August 2006: due to their large number and their different nature, these sources will be discussed in a separate paper. In Sect. 2 we describe the observations and we provide details of the data reduction. In Sect. 3 we discuss the results, which include new binaries, resolved stars, and the near-IR counterparts of two radio maser sources. We also characterize the performance of this specialized observing mode, which combines the highest angular resolution and sensitivity possible today in the near-IR. In the conclusions we describe the integration of the LO technique in the service mode operations scheme in place at the Paranal observatory and our plans for routine LO observations at that site.

\section{Observations and data analysis}

We observed a passage of the Moon in a crowded region of the Galactic bulge, in the night of March 21-22, 2006. The center of this region was located at $\approx 17^{\mathrm{h}} 42^{\mathrm{m}}$ and $-28^{\circ} 29^{\prime}$. Figure 1 shows the area, with the apparent lunar path superimposed. It can be seen that, at closest approach, the limb of the Moon as seen from Paranal reached only a few arcseconds from the true Galactic center. The region is heavily reddened by interstellar dust. A near-IR color-magnitude diagram is shown in Fig. 2. Due to extinction, few sources in this region of the sky have optical counterparts. Our LO predictions were based mostly on the 2MASS Catalog (Cutri et al. 2003). To the limit $K \leq 7.5 \mathrm{mag}$, a total of 509 sources were predicted to be occulted by the Moon in $5 \mathrm{~h}$. Our observations began at 6:11 UT and ended at 10:25 UT. A total of 51 events were recorded. The efficiency was limited in the first part of the observations by several missed or wrongly recorded events. In fact, this run represented a commissioning of the burst mode at ISAAC, which at the time had not yet been extensively tested. In the last part of the observations, with the technical aspects under control, the typical interval between recorded events was as short as three minutes and limited by the telescope pointing and data storing times.

The limit $K \leq 7.5 \mathrm{mag}$ was quite arbitrary. In fact, in the same time span, the number of occulted sources with $K \lesssim$ 11 mag was more than 15000 . As we show later, this magnitude

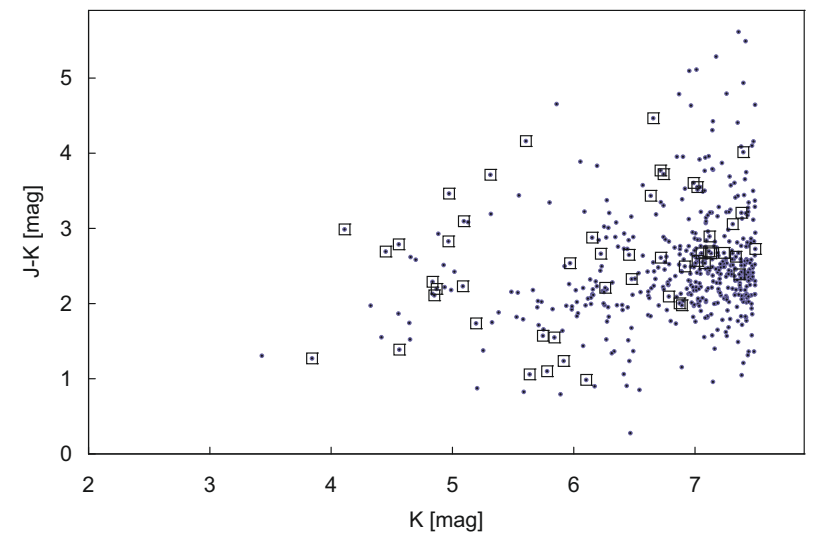

Fig. 2. Color-magnitude diagram for the sources of which we recorded LO events as listed in Table 1 (open squares). The small dots mark all sources occulted by the Moon in the same time span, to the limit $K \leq 7.5$ mag.

limit would have been realistic, but it was clearly impossible to observe hundreds, not to mention thousands, of sources. Even a manual selection of the best ones would have been a daunting task, so for this we developed a prioritization rule that combines the magnitude, the color (redder objects were given more weight), and the time intervals between the events. We also flagged and gave additional priority to sources with previous observations or classification avalailable in the SIMBAD database.

The lunar phase was $-54 \%$. In general LO observations are possible only on the dark lunar limb, thus we observed reappearances. The telescope was pointed at the nominal star position while this was still occulted, and data were recorded starting about thirty seconds before the nominal reappearance time. In practice, our predictions were accurate to within a few seconds in all cases. Thanks to the work of Paranal software engineers, the event times were included in the files that control telescope and instrument operation (so-called observing blocks or OBs). These were then loaded well in advance, and data acquisition started automatically at the preset time.

Two factors affected the quality of the resulting data significantly. First, the active optics corrections of the primary mirror had to be switched off. As a result, the image quality was rather poor especially in the first part of the run. Towards the end of the run, we activated the active optics a few times in between events, thus obtaining much better image quality. Second, in a few cases part of the signal fell outside the subwindow (clipping). Blind pointing was done by means of offsets from a nearby star, but also given the bad image quality and the small detector subwindow, it was sometimes not accurate enough. The subwindow was 

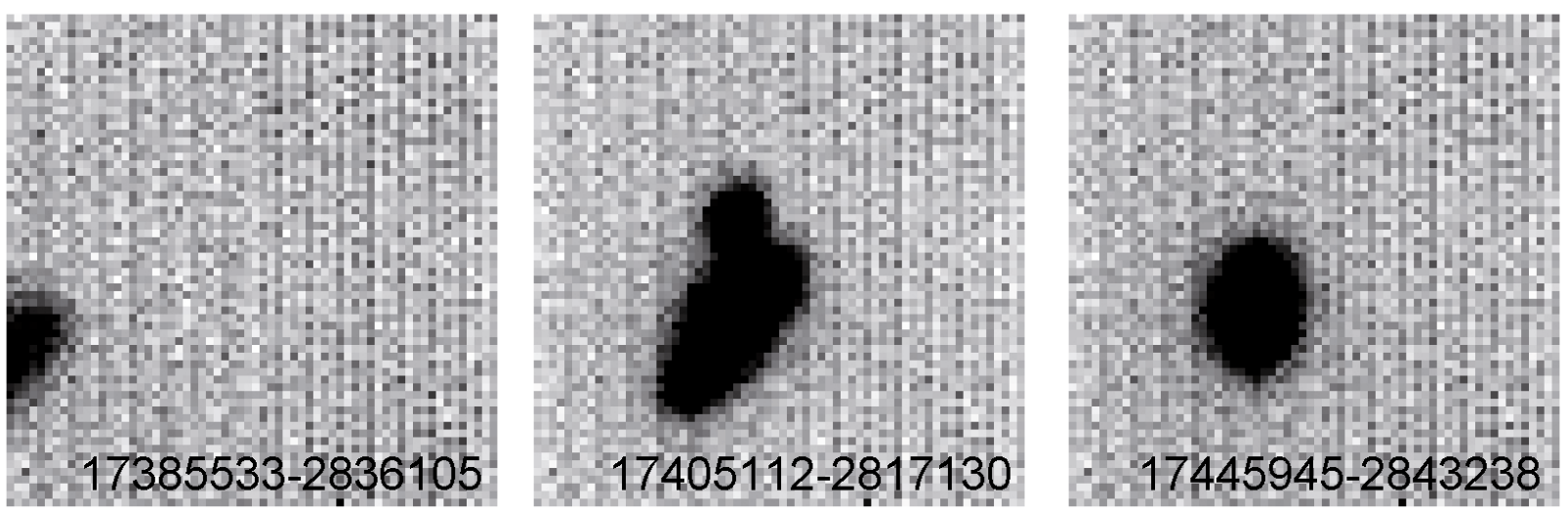

Fig. 3. Illustration of the adverse effects of image clipping due to inaccurate blind pointing (left) and of poor image quality due to the active optics being switched off (middle, note that the target is a single star), as described in the text. The right figure is an example of a more regular case, for comparison. All three figures are time averages over several thousand frames in the subwindow of the Aladdin array in ISAAC. The windows are $\approx 9.5^{\prime \prime}$ on the side. The 2MASS names of the occulted stars are indicated.

kept to $64 \times 64$ pixels, or $\approx 9.5^{\prime \prime}$ squared, in order to achieve sufficiently fast read-outs. We stress that in general this does not have too many negative effects on the occultation light curve except of course for the signal-to-noise ratio $(S N R)$. Figure 3 illustrates these effects.

The critical parameter for the angular resolution achieved in a LO, along with the $S N R$, is the sampling of the light curve. For this, the burst mode implemented on the Aladdin detector of the ISAAC instrument was the critical factor, allowing us to obtain times of just $3.2 \mathrm{~ms}$ on a $32 \times 32$ subarray and $6.4 \mathrm{~ms}$ sampling on a $64 \times 64$ subarray. The sampling time is identical to the detector integration time (DIT). To increase the accuracy of source centering during blind pointing of the occulted sources, all the events but the first two were recorded in $64 \times$ 64 mode. The length of the data stream was kept between 8000 and 14000 frames (see Table 1). We do not describe the details here of the burst mode for ISAAC, for which we refer to Richichi et al. (2008). The burst mode is also possible at several other ESO instruments at both Paranal and La Silla (see for example Domiciano de Souza et al. 2008; Poncelet et al. 2007; Sicardy et al. 2007). The raw data need to be reformatted into proper FITS files, which are then processed using the AWLORP pipeline. Schematically, this performs both an automated light curve extraction based on a mask estimation that preserves the object-pixel connectivity and computes an automated estimation of the basic parameters of each light curve, namely time of occultation, stellar signal, background signal, and rate of lunar motion. This is achieved via a novel algorithm based on wavelet decomposition. Details on AWLORP can be found in Fors (2006) and Fors et al. (2008).

Once the data are prepared with the proper structure, AWLORP runs automatically, saving the user from a lengthy and error-prone screening and producing preliminary fits and plots for all light curves. A total of 30 events were found to have data of sufficient quality: the remaining 21 were discarded because of problems in the pointing or in the data acquisition. For the data analysis, we employed the model-dependent fitting ALOR (see Richichi et al. 1996) and the model-independent estimation of the brightness profile CAL (Richichi 1989).

\section{Results}

The complete list of recorded occultation events is given in Table 1. It includes comments about the quality of the data and missed events. The $K$-band magnitudes range from 4.1 to 7.4. In the table, and throughout the paper, we use 2MASS names. Most of the sources do not have a counterpart in the Simbad database. Only 14 of the sources that were recorded reliably can be crossidentified with optical or IR sources, and these are also listed in the table.

We tested the resolved/unresolved character of all the sources, using the method first outlined by Richichi et al. (1996) and applied in some of our previous papers, such as in Fors et al. (2004) and Richichi et al. (2006a). The results are shown in Fig. 4, and we discuss below the resolved and the unresolved sources separately.

We show the complete set of light curves and fits in Fig. 9. In spite of the previously mentioned problems, we note that our sample includes some of the best LO data ever recorded, both in terms of sensitivity and of SNR. The performance of the $8.2 \mathrm{~m}$ VLT telescope for LO work proved to be outstanding, not just for its light collecting power but also for the strong reduction of atmospheric scintillation, which is often the main limiting SNR factor for LO. In terms of angular resolution, the relationship shown in Fig. 4 is consistent with that established at other, smaller telescopes for which the DIT was considerably shorter (see Richichi et al. 1996, 2006a). We note that the SNR of the fit shown in Fig. 5 is, as far as we know, the best ever achieved for a LO light curve. Just a few milliarcseconds away from the main star it would have been possible to detect a companion fainter than the primary by 7.8 and $7.1 \mathrm{mag}$ in the occulted and unocculted parts respectively, i.e. on opposite sides of the star. This compares favorably with the best AO-assisted, highcontrast imaging close to a bright star in this range of separations (Masciadri et al. 2005).

\subsection{Resolved sources}

We found two sub-arcsecond (projected) separation binaries, two stars with a convincingly resolved angular size, and three other stars that we consider as marginally resolved. These sources are listed in Table 2, using the same format already used in Richichi et al. (2006a) and other papers in that series. In summary, the columns list the absolute value of the fitted linear rate of the event $V$, its deviation from the predicted rate $V_{\mathrm{t}}$, the local lunar limb slope $\psi$, the position and contact angles, $S N R$. For binary detections, the projected separation and the brightness ratio are given, while the angular diameter $\phi_{\mathrm{UD}}$ is reported for 
Table 1. List of the recorded occultation events.

\begin{tabular}{|c|c|c|c|c|c|c|c|c|}
\hline 2MASS id & $\begin{array}{c}\text { Frame } \\
(\mathrm{px})\end{array}$ & NDIT & $\begin{array}{l}\text { Time } \\
\text { (UT) }\end{array}$ & & $I-K$ & $\overline{S p}$ & Cross-Id & Notes \\
\hline $17383314-2836158$ & 32 & 14000 & $06: 11: 57$ & 5.64 & 1.06 & - & - & df, $50 \%$ off edge \\
\hline $17383141-2812445$ & 32 & 14000 & $06: 15: 19$ & 6.78 & 2.09 & - & - & df \\
\hline 17394834-2809136 & 64 & 12000 & $06: 23: 51$ & 6.48 & 2.33 & - & - & near terminator \\
\hline $17385533-2836105$ & 64 & 12000 & $06: 28: 10$ & 7.34 & 2.62 & - & - & df, $50 \%$ off edge \\
\hline $17385407-2833227$ & 64 & 12000 & $06: 32: 56$ & 7.13 & 2.67 & - & - & df, $50 \%$ off edge \\
\hline $17391567-2826194$ & 64 & 12000 & $06: 47: 58$ & 6.46 & 2.65 & - & - & df \\
\hline $17394022-2820124$ & 64 & 12000 & 06:55:09 & 7.12 & 2.69 & - & - & df, close to edge \\
\hline $17394602-2822089$ & 64 & 12000 & 07:00:05 & 7.05 & 2.67 & - & - & df \\
\hline $17395038-2824061$ & 64 & 12000 & 07:04:00 & 5.78 & 1.10 & - & CD-28 13409 & not seen \\
\hline $17410318-2814151$ & 64 & 12000 & 07:08:12 & 7.02 & 2.57 & - & - & edge ( $75 \%$ off) \\
\hline $17402920-2842361$ & 64 & 12000 & $07: 14: 29$ & 7.50 & 2.73 & - & - & not seen \\
\hline $17405112-2817130$ & 64 & 12000 & $07: 18: 35$ & 4.87 & 2.20 & M6.5 & [RHI84] 10-333 & df \\
\hline $17412116-2816010$ & 64 & 12000 & $07: 22: 54$ & 5.84 & 1.55 & M5 & [RHI84] 10-356 & df, 2 stars \\
\hline $17402750-2833099$ & 64 & 12000 & $07: 26: 35$ & 7.24 & 2.67 & - & - & not seen \\
\hline $17411415-2818051$ & 64 & 12000 & $07: 29: 49$ & 6.87 & 2.00 & M5 & [RHI84] 10-352 & df, 2 stars \\
\hline $17404347-2827185$ & 64 & 12000 & 07:33:08 & 6.89 & 1.98 & - & - & df, $50 \%$ off edge \\
\hline $17410937-2822418$ & 64 & 12000 & $07: 39: 35$ & 6.92 & 2.49 & - & DENIS-P J174109.4-2\& & df \\
\hline $17412349-2821538$ & 64 & 12000 & $07: 44: 18$ & 7.37 & 2.39 & - & - & df \\
\hline 17411109-2840486 & 64 & 12000 & 07:47:31 & 7.31 & 3.06 & - & - & not seen \\
\hline $17423294-2818473$ & 64 & 12000 & $07: 52: 21$ & 3.84 & 1.27 & G8Iab & HD 160706 & near terminator \\
\hline $17414641-2822593$ & 64 & 12000 & 07:57:10 & 5.09 & 2.23 & - & ISOGAL-P J174146.5-282301 & $\mathrm{df}$ \\
\hline $17413435-2829225$ & 64 & 12000 & 08:01:38 & 4.85 & 2.11 & - & DENIS-P J174134.4-282922 & df, $10 \%$ off edge \\
\hline $17414185-2842287$ & 64 & 12000 & 08:05:22 & 6.22 & 2.66 & - & - & not seen \\
\hline $17415470-2826596$ & 64 & 12000 & 08:09:09 & 7.08 & 2.54 & - & DENIS-P J174154.7-282659 & df, $95 \%$ off edge \\
\hline $17425620-2820370$ & 64 & 12000 & $08: 13: 32$ & 4.56 & 1.39 & - & 2MASS J17425620-2820370 & not seen \\
\hline $17415719-2834236$ & 64 & 12000 & 08:17:55 & 7.12 & 2.89 & - & - & very faint \\
\hline $17420509-2833465$ & 64 & 12000 & $08: 22: 10$ & 6.15 & 2.88 & - & - & df, $50 \%$ off edge \\
\hline $17423746-2825311$ & 64 & 12000 & $08: 27: 23$ & 5.20 & 1.74 & - & - & df, very faint \\
\hline $17424039-2826255$ & 64 & 12000 & 08:31:04 & 4.56 & 2.79 & - & IRAS 17395-2825 & not seen \\
\hline $17432345-2853503$ & 64 & 12000 & $08: 38: 21$ & 4.97 & 3.46 & - & ISOGAL-P J174323.6-285349 & not seen \\
\hline $17432585-2823598$ & 64 & 12000 & 08:44:05 & 5.92 & 1.24 & - & - & df, $15 \%$ off edge \\
\hline $17430791-2828016$ & 64 & 12000 & 08:48:53 & 7.02 & 3.55 & - & - & df \\
\hline $17425497-2836593$ & 64 & 12000 & 08:53:18 & 6.72 & 3.77 & - & - & df, $50 \%$ off edge \\
\hline $17432661-2827590$ & 64 & 12000 & 08:58:32 & 6.26 & 2.21 & M2: & [RHI84] 10-443 & df, $50 \%$ off edge \\
\hline $17430901-2837039$ & 64 & 12000 & 09:01:53 & 6.99 & 3.60 & - & - & df, $30 \%$ off edge \\
\hline 17431348-2837004 & 64 & 12000 & 09:04:34 & 6.74 & 3.72 & - & - & df \\
\hline $17434830-2828129$ & 64 & 12000 & 09:10:32 & 4.84 & 2.29 & - & - & df, $95 \%$ off edge \\
\hline 17435893-2827521 & 64 & 12000 & 09:15:00 & 5.97 & 2.54 & - & - & df, second star \\
\hline $17444369-2825027$ & 64 & 12000 & 09:17:24 & 7.38 & 3.21 & - & - & df, $5 \%$ off edge \\
\hline 17435704-2830432 & 64 & 12000 & 09:21:47 & 5.61 & 4.16 & - & IRAS 17407-2829 & df, $25 \%$ off edge \\
\hline $17434681-2840176$ & 64 & 12000 & 09:26:43 & 4.45 & 2.69 & M6.5 & ISOGAL-P J174346.6-284017 & not seen \\
\hline 17435399-2841285 & 64 & 12000 & $09: 31: 28$ & 4.11 & 2.99 & - & IRAS 17407-2840 & df \\
\hline $17445251-2826479$ & 64 & 12000 & 09:36:45 & 5.09 & 3.10 & - & - & not seen \\
\hline 17441366-2848501 & 64 & 8000 & 09:39:53 & 6.10 & 0.99 & K0 & HD 316221 & $5 \%$ off edge \\
\hline $17443976-2830568$ & 64 & 10000 & 09:46:46 & 7.40 & 4.01 & - & - & not seen \\
\hline $17452448-2826434$ & 64 & 12000 & 09:49:33 & 6.72 & 2.61 & - & - & not seen \\
\hline 17450413-2853412 & 64 & 12000 & 10:05:20 & 5.75 & 1.57 & M2 & [RHI84] 10-515 & df \\
\hline $17452968-2829325$ & 64 & 12000 & 10:10:43 & 6.64 & 3.43 & - & - & gf, 2 more stars \\
\hline $17445945-2843238$ & 64 & 12000 & 10:14:15 & 4.97 & 2.83 & - & - & gf \\
\hline $17460150-2828035$ & 64 & 12000 & $10: 19: 24$ & 6.66 & 4.47 & - & - & gf \\
\hline $17453224-2833429$ & 64 & 12000 & $10: 25: 26$ & 5.31 & 3.71 & - & ISOGAL-P J174532.3-283338 & gf, $5 \%$ off edge \\
\hline
\end{tabular}

Frame is the size of the subwindow and NDIT the number of frames used in the raw burst mode. The number of frames in the reformatted FITS cube is NDIT/2-1, and this also determines the total time span of the recorded data. In the notes, df stands for defocused and gf for good focus.

resolved stars, under the assumption of a uniform stellar disk. All angular quantities are computed from the fitted rate of the event.

Several of our acquisition images show companions, but we only report two binaries, which have projected separations below one arcsecond. This is rather large for the context of LO binary detections. In fact, for separations $\gtrsim 0$ '. 5 , the $\mathrm{LO}$ technique is relatively inaccurate, due to possible differences in the local limb slope at the points of occultation of the two components. The two objects are 2MASS 17412116-2816010 and 2MASS 174443692825027. They are most likely projection and not physical pairs, but we report them here for completeness, since no previous mention exists in the literature. The former was classified by Raharto et al. (1984) as an M5 star. We note that the difference between predicted and observed lunar rates for the second star was quite significant, as reported in Table 2 . The contact angle for this event was only $260^{\circ}$, i.e. nearly grazing, and the predicted lunar rate only -0 . $^{\prime} 1 / \mathrm{s}$. Under these conditions, it is 
Table 2. Summary of results.

\begin{tabular}{|c|c|c|c|c|c|c|c|c|c|}
\hline (1) & (2) & (3) & (4) & $\overline{\overline{(5)}}$ & $\overline{(\overline{(6)})}$ & $\overline{(\overline{(7)}}$ & $\overline{(8)}$ & 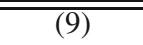 & (10) \\
\hline Source & $|V|(\mathrm{m} / \mathrm{ms})$ & $V / V_{\mathrm{t}}-1$ & $\psi\left(\left(^{\circ}\right)\right.$ & $\mathrm{PA}\left({ }^{\circ}\right)$ & $\mathrm{CA}\left({ }^{\circ}\right)$ & $S N R$ & Sep. (mas) & Br. Ratio & $\phi_{\text {UD }}$ (mas) \\
\hline $17391567-2826194$ & 0.7702 & $-2.9 \%$ & -7 & 268 & 163 & 211.0 & & & $2.68 \pm 0.66$ \\
\hline $17412116-2816010$ & 0.3245 & $5.7 \%$ & -2 & 350 & 245 & 105.4 & $711.7 \pm 3.4$ & $22.6 \pm 0.8$ & \\
\hline $17435893-2827521$ & 0.4688 & $4.9 \%$ & -3 & 321 & 222 & 233.2 & & & $2.22 \pm 0.37$ \\
\hline $17444369-2825027$ & 0.1802 & $62.3 \%$ & & see text & & 68.2 & $171.6 \pm 25.8$ & $47.8 \pm 7.5$ & \\
\hline $17435399-2841285$ & 0.6095 & $-1.4 \%$ & -5 & 266 & 168 & 460.0 & & & $2.36 \pm 0.11$ \\
\hline $17445945-2843238$ & 0.5945 & $-1.7 \%$ & -7 & 263 & 168 & 251.4 & & & $1.91 \pm 0.30$ \\
\hline $17453224-2833429$ & 0.5328 & $7 \%$ & -6 & 302 & 209 & 126.9 & & & 2.9 (shell) \\
\hline
\end{tabular}

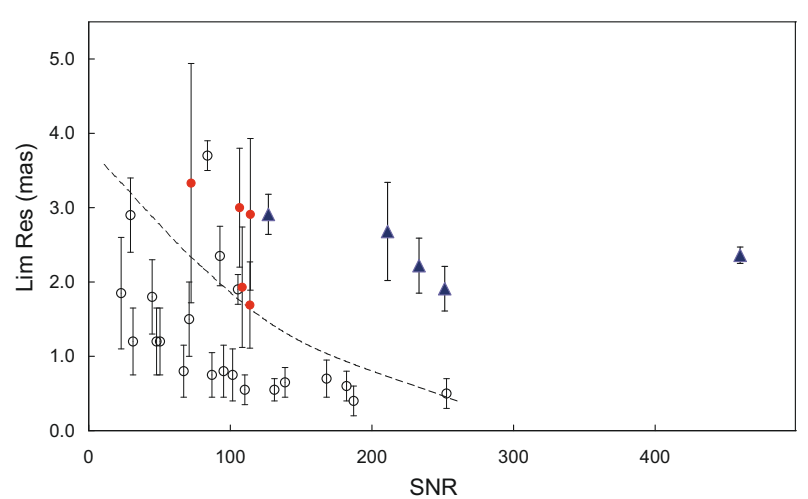

Fig. 4. Sources for which an upper limit on the angular diameter could be established using the method described in the text (marked with outlined circles). The filled circles are sources for which an angular diameter could be formally established, but for which the associated errorbar is such that they are consistent with being unresolved. The dashed line is an arbitrary representation of a tentative SNR-limiting angular resolution relationship. The triangles are the sources which we consider tentatively or convincingly resolved.

common that either a small error in the predictions or a local limb slope can significantly alter the computation of the effective PA and CA.

The remaining stars in Table 2 are those appearing significantly different from point-like. The two most interesting ones are 2MASS 17435399-2841285 and 2MASS 174532242833429, which happen to both be masing AGB stars. Unfortunately, the distance determination for these stars is uncertain, especially in the absence of detailed photometric and pulsational properties. The former has an angular size that, albeit small in absolute terms, appears to be convincingly established (see Figs. 4 and 5). This star is cross-identified with IRAS 17407-2840, an $\mathrm{SiO}$ maser previously measured by Messineo et al. (2002, 2004, 2005). Its radial velocity is negative and cannot be used for a kinematic distance, but Messineo (priv. comm.) estimates a distance of $3.7 \mathrm{kpc}$ based on the average extinction and the model of Drimmel (2003). According to this, the angular size listed in Table 2 would translate to over 8 AU. Apart from consideration of the distance uncertainty, it is likely that the LO-derived size is not indicative of an uniform-disk diameter but rather a mix of the photosphere and of the immediate circumstellar surroundings.

2MASS 17453224-2833429 has a similar nature and set of references, with the addition of Sjouwerman et al. (2004). The fit for this source gave a $S N R$ of 127 which is lower than expected for the magnitude of this source $(K=5.1 \mathrm{mag})$, and the UD size reported in Table 2 should only be considered as an indication. A model-independent analysis by CAL revealed a faint but well-defined extended emission around the central component,

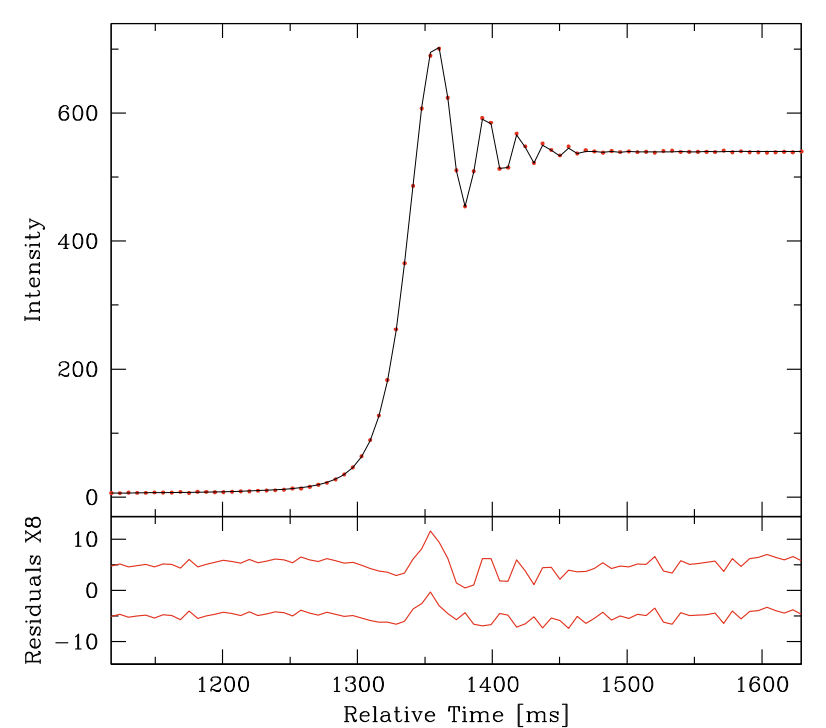

Fig. 5. Top: data (dots) and best fit (solid line) for 2MASS 174353992841285. Bottom: enlarged by eight and displaced by arbitrary offsets for clarity, the residuals of the fits with a point-like source (above, reduced $\chi^{2}=2.0$ ) and with a uniform-disk model (below, reduced $\left.\chi^{2}=1.1\right)$.

as shown in Fig. 6. The profile of this emission is almost symmetrical, has the rim brightening typical of optically thin shells, and is consistent with the masing nature of this star. In this case, both a kinematic and an extinction distance can be estimated, at 4.5 and $3.3 \mathrm{kpc}$, respectively (Messineo, priv. comm.). By using their average, the inner shell radius of 10-15 mas would translate to $\approx 40 \mathrm{AU}$. We note that the residuals of the uniform-disk model for 2MASS 17435399-2841285 indicate the possibility of a circumstellar shell also being detected in this case (cf. Fig. 5); but given that the reduced $\chi^{2}$ was already significantly good, we did not proceed with more extreme fits. The different signatures of the shell for these two maser stars could be related to their difference in $J-K$ color (3.0 versus $3.7 \mathrm{mag}$ ).

The remaining three stars in Table 2 appear resolved, although their location above the $S N R$-resolution relationship of Fig. 4 is less definite than for the two maser stars just mentioned and we prefer to consider their diameters as tentative for the moment. There are no known cross-identifications or literature entries for these stars.

\subsection{Unresolved stars and performance}

Most of the stars in our sample appear unresolved, as is to be expected from random observations of field stars. Richichi et al. (2006a) discussed the incidence of binary stars in such samples for LO observations based on the 2MASS catalog, and our 


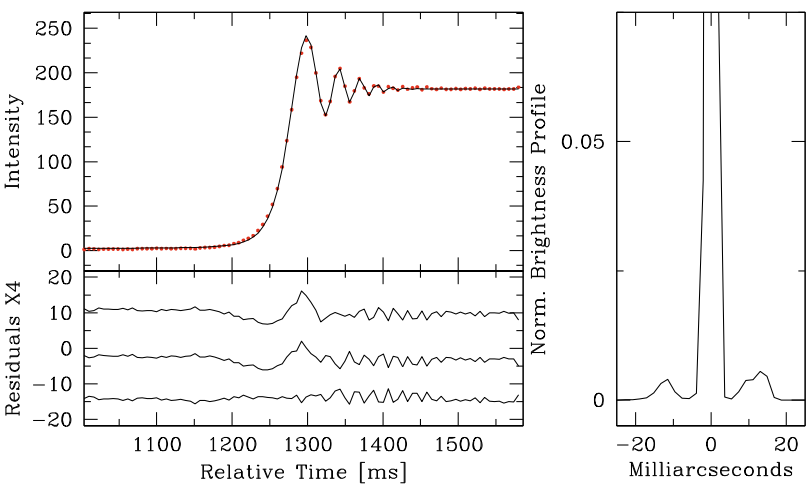

Fig. 6. Left: upper panel, data (dots) and best fit (solid line) for 2MASS 17453224-2833429. The lower panel shows, on a scale enlarged by four and displaced by arbitrary offsets for clarity, the residuals of three different fits (reduced $\chi^{2}=6.3$ ). Bottom, fit with the modelindependent CAL method (reduced $\chi^{2}=1.6$ ). Right: brightness profile reconstructed by the CAL method.

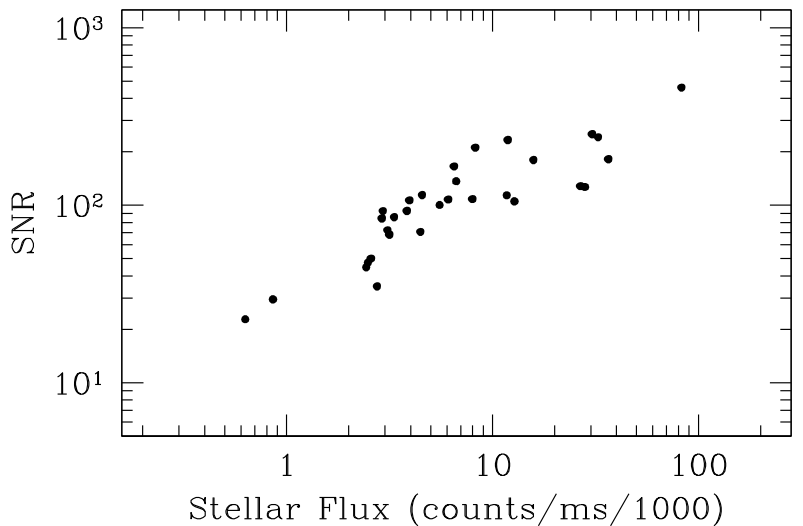

Fig. 7. Plot of the $S N R$ achieved in our fits of both resolved and unresolved source, as a function of the total measured flux.

present results are consistent within the uncertainty of small statistics. The unresolved stars are also useful, namely in that the relationship of Fig. 4 establishes rather strict upper limits on their size and on the brightness ratio of unseen components, if any. Thus these stars can be safely used as calibrators for longbaseline interferometry.

Furthermore, the unresolved stars allow us to investigate the performance of the burst mode at the VLT for LO, similar to what was done for other instrument and telescope configurations in the past (see Fors et al. 2004). In Fig. 7 we plot the SNR achieved in the fits of all sources (the unresolved ones being the majority), against the total measured flux. This is a better quantity to use than the actual magnitude, since as mentioned earlier, many of our sources were recorded at the edge of the subarray field with considerable loss of signal. Also intrinsic variability might be the cause for some of the stars. Indeed, Fig. 8 shows that many outliers from the ideal $K$ magnitude-observed flux are present. Using the measures signal instead, Fig. 7 shows a rather linear relationship across over two orders of magnitudes in flux. This implies that, at the faint end, we were still in a photonlimited regime, and at at the bright end, the incidence of scintillation and possible nonlinearities was not yet significant. We estimate that nonlinearity of the detector should become important above $K \approx 3 \mathrm{mag}$, i.e. one magnitude above our brightest target for this run.

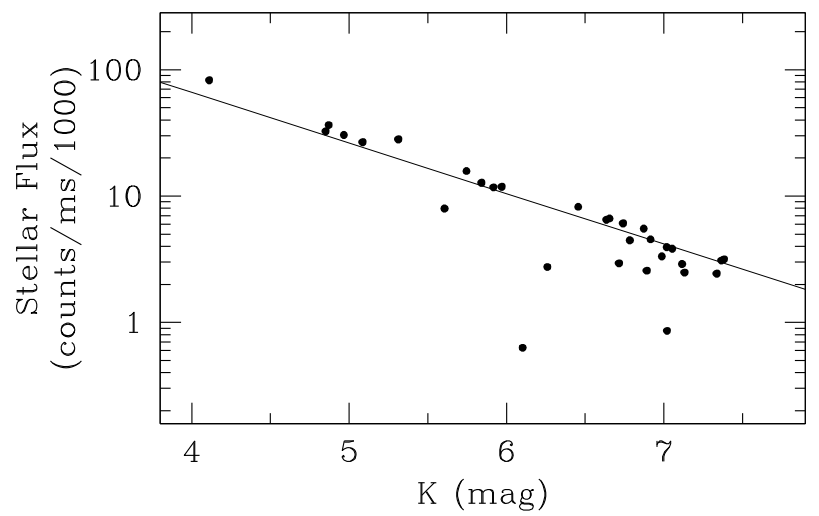

Fig. 8. Plot of the total measured flux against the $K$ magnitude of the sources (from 2MASS). The line shows the expected counts, based on the ISAAC Exposure Time Calculator. Note the discrepant cases, due to the problems mentioned in the text.

In spite of the outliers, the relationship between $K$ magnitude and counts clearly emerges from Fig. 8, and it is in excellent agreement with the expected ISAAC performance. Comparing the two figures, we estimate at first approximation that under the same conditions of sampling and DIT $(6.4 \mathrm{~ms})$, it should have been possible to obtain $S N R=1$ for $K \approx 12 \mathrm{mag}$. This is the minimum $S N R$ needed to detect a faint companion in a LO light curve, although measurements of angular diameters or extended emission would require $S N R \gtrsim 10$. At the faint magnitude end, critical parameters are the diffuse scattered light background, which is a function of the lunar phase and distance of the occultaton point from the terminator and the pixel scale. Ideally, the latter should match the seeing disk, but in our case with a pixel scale of about $0 . ' 148$, we significantly oversampled the background.

\section{Conclusions}

We have presented the first LO results obtained using the socalled burst mode of the ISAAC instrument at the ESO Antu VLT telescope. This mode permits data streams to be recorded on a small subarray at high temporal frequency $(3.2 \mathrm{~ms}$, although mostly $6.4 \mathrm{~ms}$ were used for the present work). We recorded 51 occultation events over about four hours during a close approach of the Moon to the Galactic center. The events were reappearances from behind the dark limb. This, coupled with initial problems of focusing and image quality due mostly to the commissioning nature of the run, resulted in several sources being missed or without sufficient quality. Nevertheless, 30 of the events resulted in light curves of good, or even excellent quality, including some of the best $S N R$ traces ever recorded with this technique. We have revealed two binaries, three stars with a marginally resolved angular diameter on the order of 2 mas, and two resolved masing AGB stars which appear to be in the foreground of the Galactic bulge. For one of these, 2MASS 17453224-2833429, we were able to recover the brightness profile of the extended emission. This has the typical signature of an optically thin shell, with inner radius of about 10-15 mas, corresponding to about 40 AU. Follow-up studies are required to obtain further information on these stars, given that they are all heavily reddened and most have no known counterpart or literature entries.

Our analysis, including the unresolved sources, has established an unprecedented performance of the LO technique in the near-IR using a very large telescope and a fast read-out mode. 

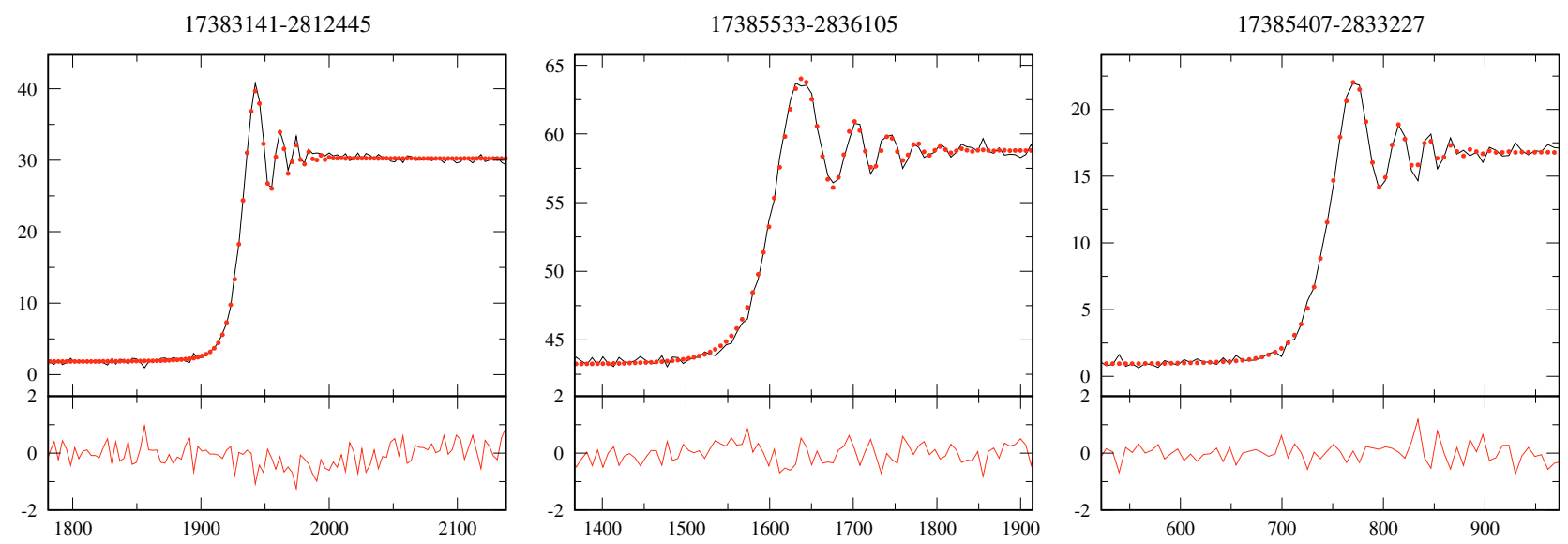

17391567-2826194

17394022-2820124

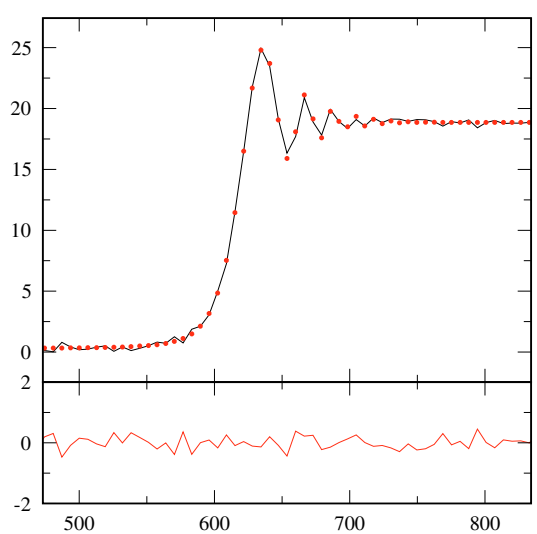

17405112-2817130

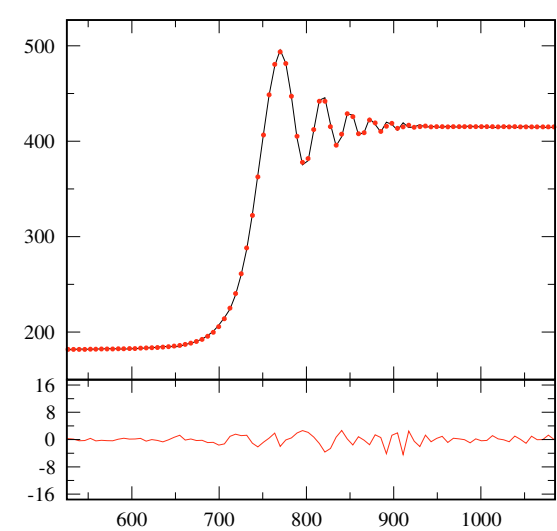

17404347-2827185

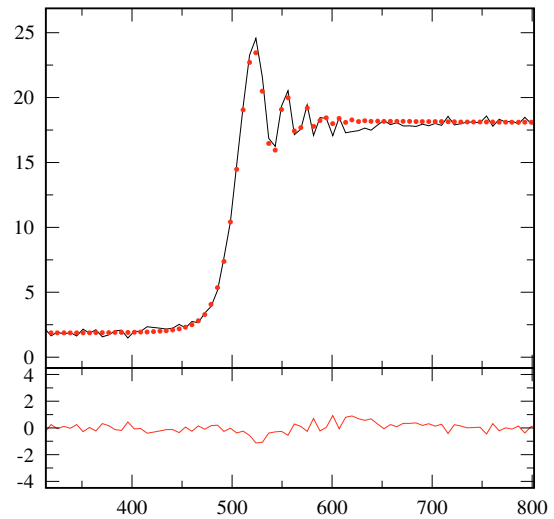

17394602-2822089

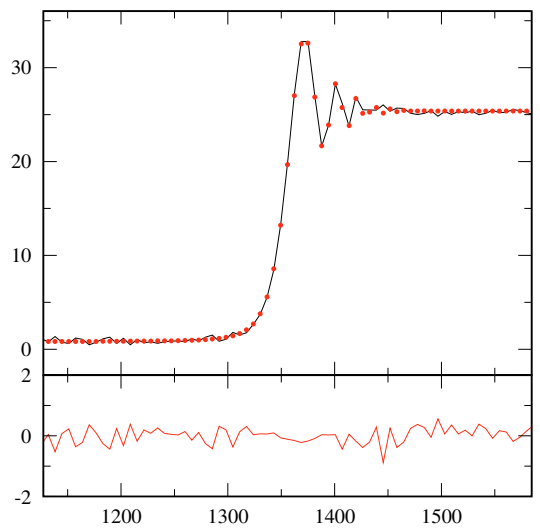

17412116-2816010

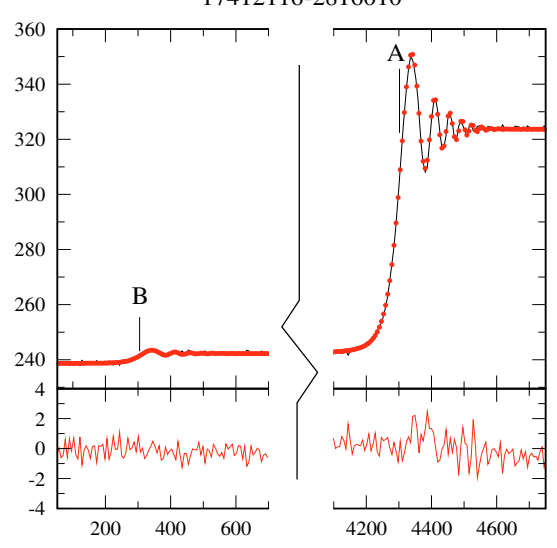

$17410937-2822418$

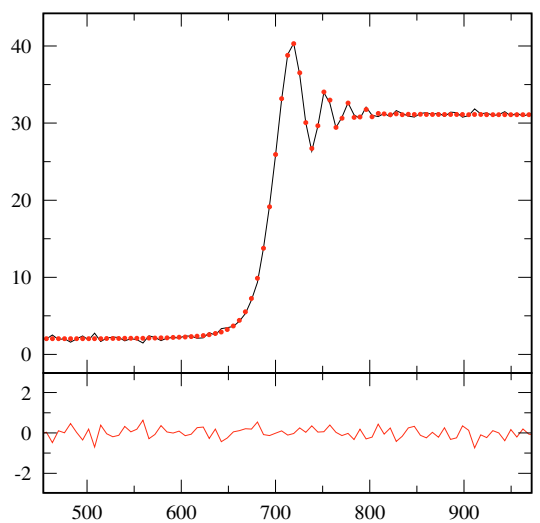

Fig. 9. Top panels: observed LOs and best fits. Bottom panels: fit residuals on an enlarged scale. 

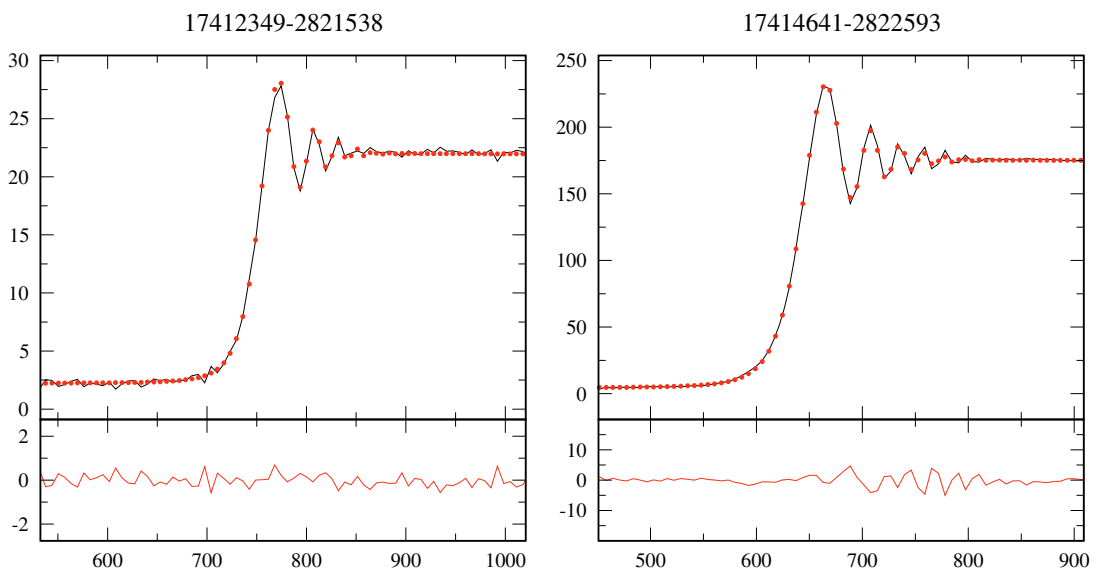

$17413435-2829225$

17432585-2823598

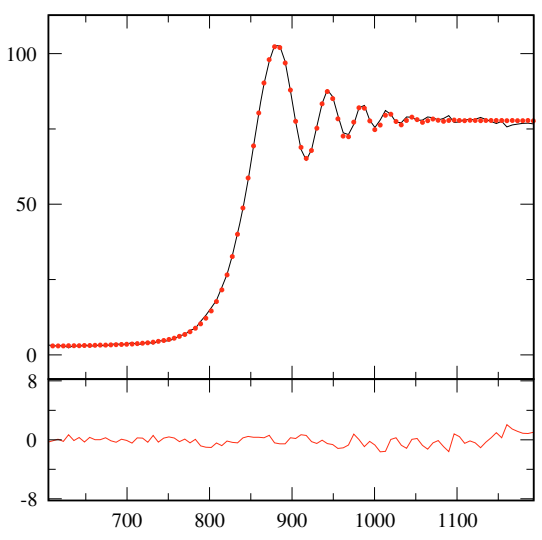

17430791-2828016
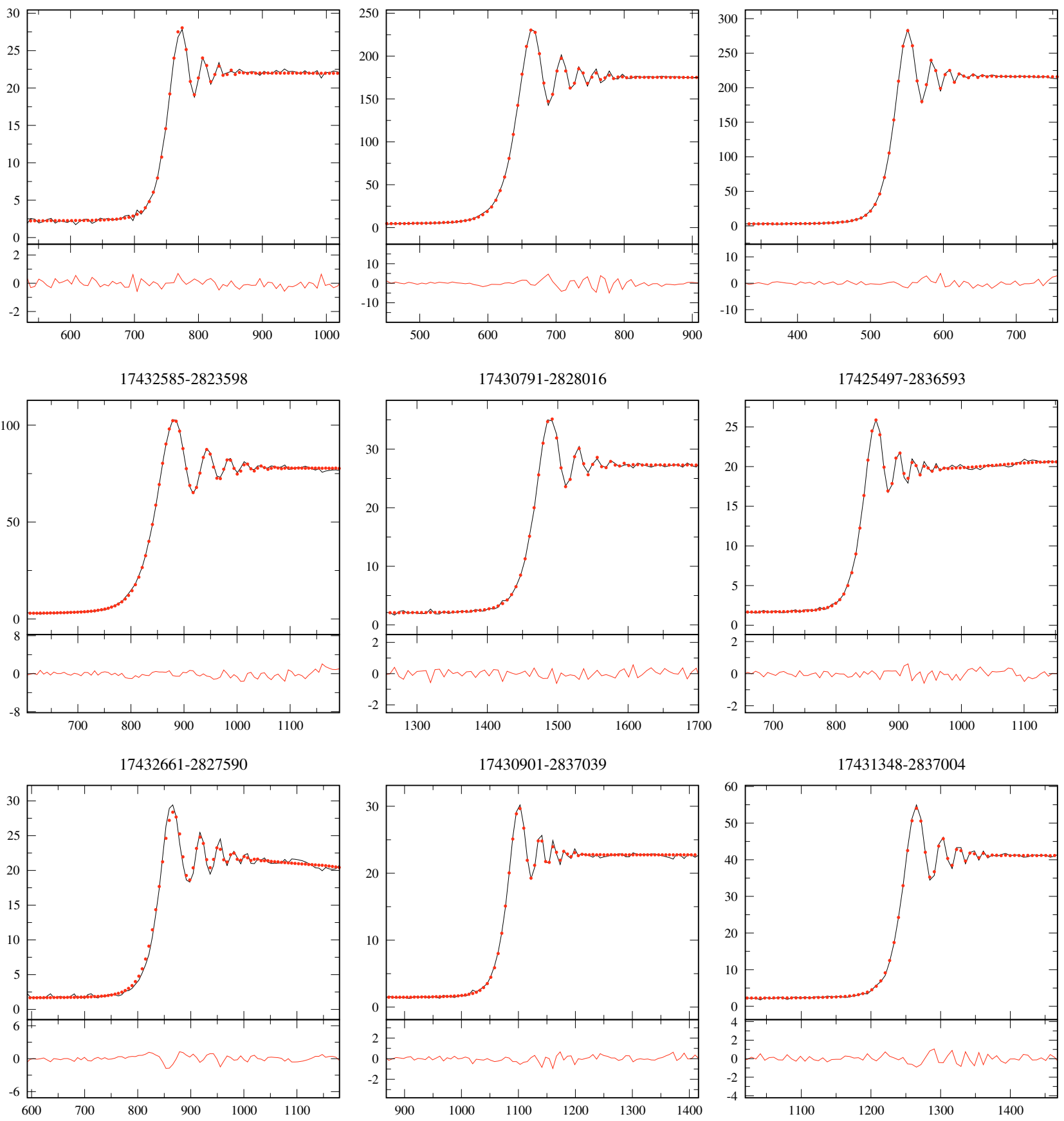

17435893-2827521

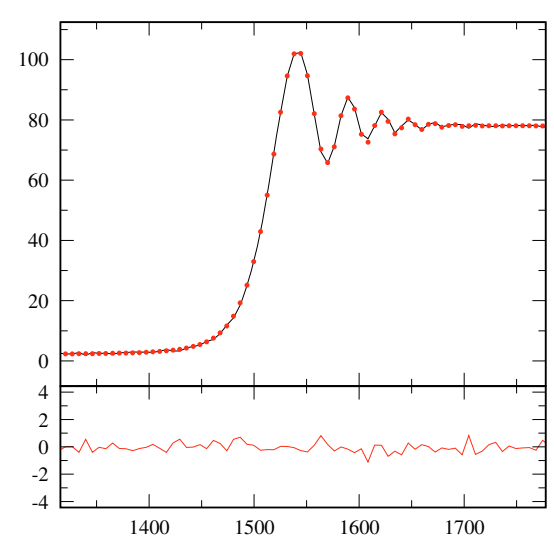

17444369-2825027

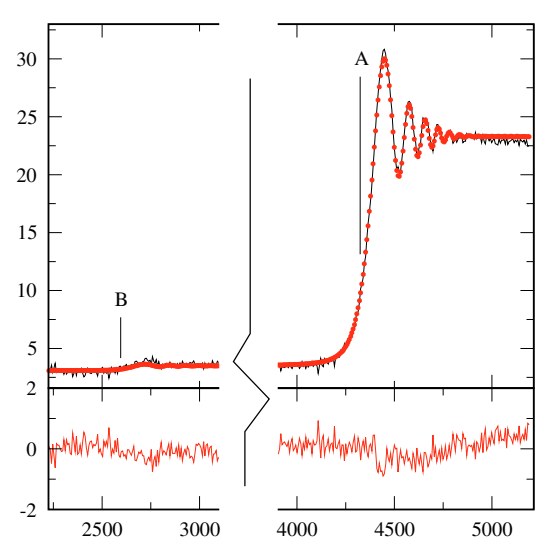

17435704-2830432

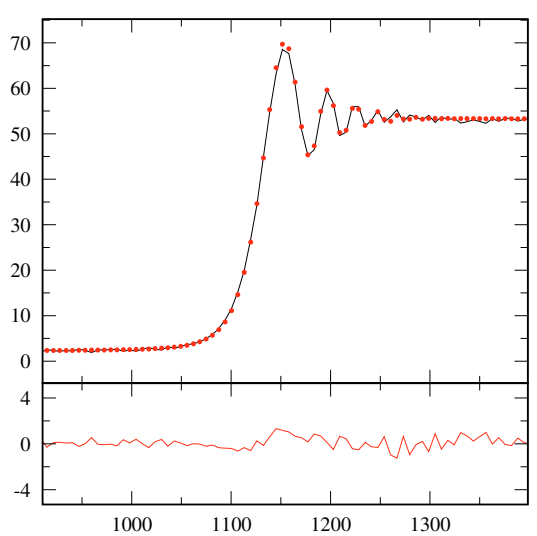

Fig. 9. continued. 
$17435399-2841285$

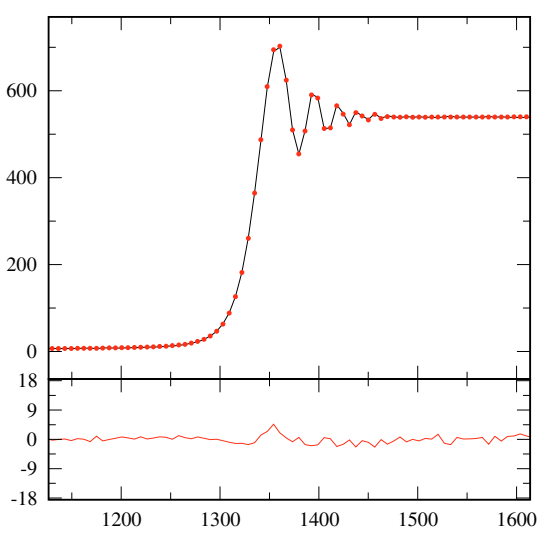

$17452968-2829325$

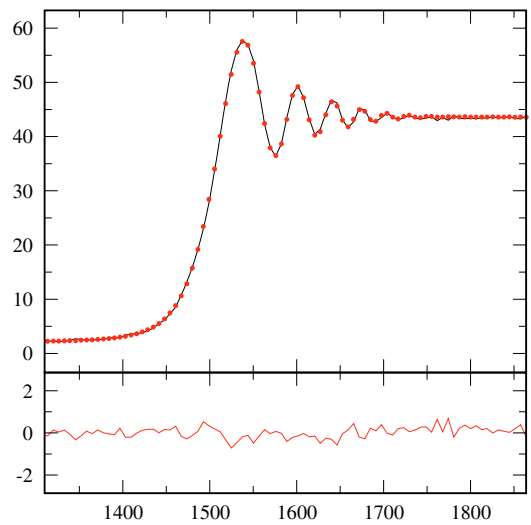

$17453224-2833429$

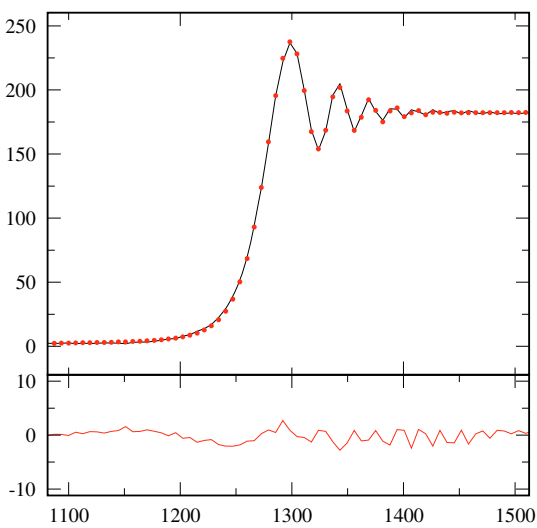

$17441366-2848501$

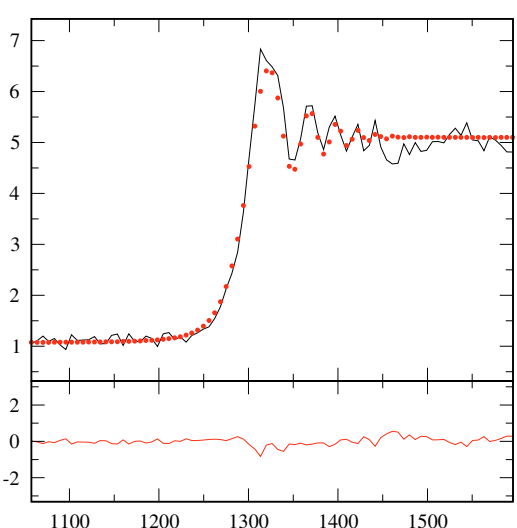

$17445945-2843238$

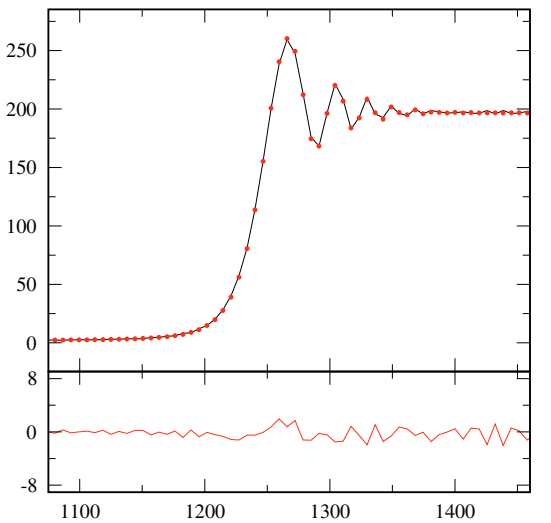

$17450413-2853412$

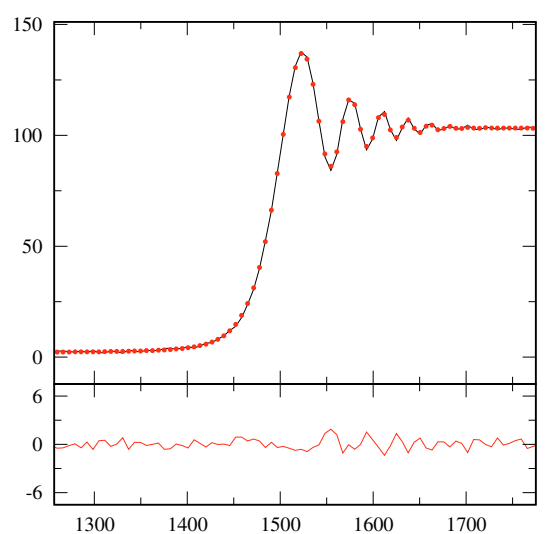

$17460150-2828035$

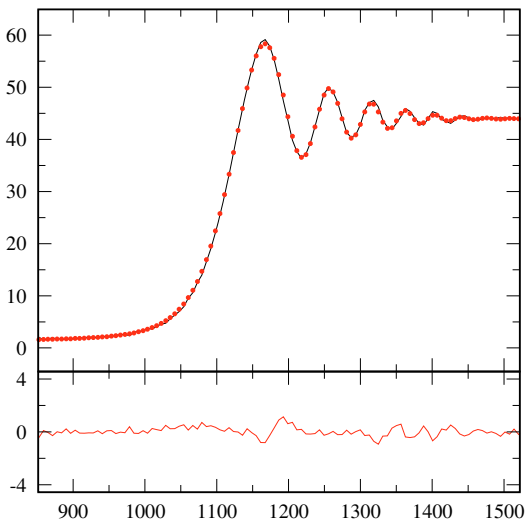

Fig. 9. continued.

We find that the angular resolution varies between 4 and 1 mas, and even less in the case of very high $S N R$. Data quality is generally impressive, compared to smaller telescopes, mostly due to the scintillation reduction effect of the large mirror. The dynamic range is also significantly improved over previous observations, and we have shown that in some cases it would have been possible to detect a companion fainter than the primary by about $8 \mathrm{mag}$ in broad $K$ band on angular scales comparable to the Airy disk of the telescope. This result compares favorably with other AO-assisted, high-contrast imaging techniques.

The random selection of sources to be occulted and the fixed-time nature of the events remain the main downsides of the LO technique. However, thanks to the very short telescope time required for each observation, LO are an ideal filler program for times when the telescope is idle or other programs cannot be conveniently executed. The service mode observations implemented at the VLT provide the right context for this, and we have obtained approval for such a filler program during ESO observing periods 80 and 81 (from October 2007 through September 2008). Several hundred OBs have been prepared for each period and are awaiting execution whenever five minutes of telescope time become available.

Acknowledgements. This work is partially supported by the ESO Director General's Discretionary Fund and by the MCYT-SEPCYT Plan Nacional $I+D+I$ AYA \#2005-08604. A.R. wishes to thank the National Astronomical Observatory 
of Japan in Mitaka, and Dr. J. Nishikawa in particular, for their hospitality during a prolonged stay, which led to the final writing of this paper. We thank Dr. M. Messineo for private communications on the properties of the $\mathrm{SiO}$ masers reported in this paper. This research made use of the SIMBAD database, operated at the CDS, Strasbourg, France.

\section{References}

Cutri, R. M., Skrutskie, M. F., van Dyk, S., et al. 2003, The IRSA 2MASS AllSky Point Source Catalog, NASA/IPAC Infrared Science Archive

Domiciano de Souza, A., Kervella, P., Bendjoya, P., \& Niccolini, G. 2008, A\&A, 480, L29

Drimmel, R., Cabrera-Lavers, A., \& López-Corredoira, M. 2003, A\&A, 409, 205

Fors, O. 2006, Ph.D. Thesis, Departament d'Astronomia i Meteorologia, Universitat de Barcelona

Fors, O., Richichi, A., Núñez, J., \& Prades, A. 2004, A\&A, 419, 285

Fors, O., Richichi, A., Mason, E., Stegmeier, J., \& Chandrasekhar, T. 2006, Highlights of Spanish Astrophysics IV, ed. Figueras et al. (Springer)

Fors, O., Richichi, A., Otazu, X., \& Nuñez, J. 2008, A\&A, 480, 297

Masciadri, E., Mundt, R., Henning, T., Alvarez, C., \& Barrado y Navascués, D. 2005, ApJ, 625, 1004
Messineo, M., Habing, H. J., Sjouwerman, L. O., Omont, A., \& Menten, K. M. 2002, A\&A, 393, 115

Messineo, M., Habing, H. J., Menten, K. M., Omont, A., \& Sjouwerman, L. O. 2004, A\&A, 418, 103

Messineo, M., Habing, H. J., Menten, K. M., et al. 2005, A\&A, 435, 575

Poncelet, A., Doucet, C., Perrin, G., Sol, H., \& Lagage, P. O. 2007, A\&A, 472, 823

Raharto, M., Hamajima, K., Ichikawa, T., Ishida, K., \& Hidayat, B. 1984, Ann. Tokyo Astron. Obs., 19, 469

Richichi, A. 1985, Thesis, Faculty of Physics, Florence University

Richichi, A. 1989, A\&A, 226, 366

Richichi, A. 1997, in Very High Angular Resolution Imaging, IAU Symp., 158, 71

Richichi, A., Baffa, C., Calamai, G., \& Lisi, F. 1996, AJ, 112, 2786

Richichi, A., Percheron, I., \& Khristoforova, M. 2005, A\&A, 431, 773

Richichi, A., Fors, O., Merino, M., et al. 2006a, A\&A, 445, 1081

Richichi, A., Fors, O., Mason, E., \& Stegmeier, J. 2006b, The Messenger, 126, 24

Richichi, A., Fors, O., Mason, E., \& Stegmeier, J. 2008, Proc. of the ESO Workshop Science with the VLT in the ELT Era, ed. A. Moorwood, in press Sicardy, B., et al. 2007, AAS/Division for Planetary Sciences Meeting Abstracts, 39, \#62.02

Sjouwerman, L. O., Messineo, M., \& Habing, H. J. 2004, PASJ, 56, 45 